\title{
In vivo expression patterns of microRNAs of Gallid herpesvirus 2 (GaHV-2) during the virus life cycle and development of Marek's disease lymphomas
}

\author{
Pu Zhao $\cdot$ Xiu-Jie Li $\cdot$ Man Teng $\cdot$ Lu Dang $\cdot$ \\ Zu-Hua Yu $\cdot$ Jia-Qi Chi · Jing-Wei Su • \\ Gai-Ping Zhang • Jun Luo
}

Received: 17 September 2014 / Accepted: 7 January 2015/Published online: 11 February 2015

(c) The Author(s) 2015. This article is published with open access at Springerlink.com

\begin{abstract}
In the past decade, a large number of microRNAs (miRNAs) have been identified in the viral genome of Gallid herpesvirus 2 (GaHV-2), which is historically known as Marek's disease virus type 1 . The biological role of most GaHV-2 miRNAs remains unclear. In the present study, we have performed an overall gene expression profile of GaHV-2 miRNAs during the virus life cycle at each phase of the developing disease, a highly contagious, lymphoproliferative disorder, and neoplastic immunosuppressive disease of poultry known as the Marek's disease. According to their distinct in vivo expression patterns, the GaHV-2 miRNAs can be divided into three groups: 12 miRNAs in group I, including miR-M4-5p, displayed a typical expression pattern potentially correlated to the latent, late cytolytic, and/or the proliferative phases in the cycle of GaHV-2 pathogenesis; group II consisting of another 12 miRNAs with expression correlated to the early cytolytic and/or latent phases in GaHV-2's life cycle; while the other two miRNAs in group III showed no
\end{abstract}

Edited by Stacey Schultz-Cherry.

P. Zhao $\cdot$ L. Dang

College of Veterinary Medicine, Northwest A\&F University,

Yangling 712100, People's Republic of China

P. Zhao - X.-J. Li · M. Teng - L. Dang - Z.-H. Yu · J.-Q. Chi ·

J. Luo $(\square)$

Key Laboratory of Animal Immunology of the Ministry of Agriculture, Henan Provincial Key Laboratory of Animal Immunology, Henan Academy of Agricultural Sciences, No. 116 Huayuan Road, Zhengzhou 450002, People's Republic of China e-mail: luojun593@aliyun.com

P. Zhao

Department of Animal Science and Technology, He'nan Institute of Science and Technology, Xinxiang 453003,

People's Republic of China identical expression features. Our findings may provide meaningful clues in the search for further potential functions of viral miRNAs in GaHV-2 biology.

Keywords MicroRNA - GaHV-2 - MDV-1 - Expression profile $\cdot$ Virus life cycle $\cdot$ Oncogenesis

Gallid herpesvirus 2 (GaHV-2), commonly known as Marek's disease virus type 1 (MDV-1), is an oncogenic avian herpesvirus and classified as a member of the subfamily Alphaherpesvirinae [1]. GaHV-2 establishes and maintains latent infections in their natural hosts and may finally leads to a rapid-onset aggressive $\mathrm{T}$ cell lymphoma, an important neoplastic immunosuppressive disease of poultry named as Marek's disease (MD) [2]. GaHV-2 infection provides an ideal model for investigating the biology, genetics, and immunology of virally induced tumors [3]. In the last decade, over 400 viral microRNAs (miRNAs) have been identified in diverse virus families [4], in particular those of the herpes viruses [5, 6], some of

Present Address:

Z.-H. Yu

College of Animal Science and Technology, Henan University

of Science and Technology, Luoyang 471003,

People's Republic of China

J.-W. Su · G.-P. Zhang ( $\varangle)$

College of Animal Science and Veterinary Medicine,

Henan Agricultural University, No. 63 Nongye Road,

Zhengzhou 450002, People's Republic of China

e-mail: zhanggaiping2003@163.com

G.-P. Zhang

Jiangsu Co-innovation Center for Prevention and Control of Important Animal Infectious Diseases and Zoonoses,

Yangzhou 225009, People's Republic of China 
which have been demonstrated to potentially contribute to the control of lysis and latency, immune evasion, cell survival, proliferation, and oncogenic capabilities. Recently, a large numbers of miRNAs, which show a highly conserved genomic location, have been identified in the long or short repeat regions $\left(\mathrm{R}_{\mathrm{L}} / \mathrm{R}_{\mathrm{S}}\right)$ of the viral genomes of GaHV-2, Gallid herpesvirus 3 (GaHV-3), and Meleagrid herpesvirus 1 (MeHV-1) [7-11].

In GaHV-2 genomes, a total of 26 miRNAs are encoded in 14 precursors and concentrated in three gene clusters, namely the Meq-cluster, the LAT-cluster, and the Mid-cluster [7, 8, 12], with their transcription driven by two distinct promoters $[13,14]$. It is known that in miRNA biogenesis $[15,16]$, the primary miRNAs (pri-miRNAs) are mostly transcribed in a typical Pol II-dependent manner, to be processed into precursor miRNAs (pre-miRNAs). The precursors are transported from the nucleus to the cytoplasm, where they are cleaved by Dicer to produce the RNA duplexes, of which the functional miRNA is stably incorporated into an RNA-induced silencing complex (RISC) to regulate gene expression post-transcriptionally. Primary studies on GaHV-2 miRNAs have shown that most are expressed at higher levels in tumors and virally transformed T-lymphoma cell lines than in virusinfected chicken embryo fibroblast (CEF) [7, 8]. The miRNA of oncogenic GaHV-2 strains of differing virulence has highly conserved sequences, and the Meq-clustered miRNAs are more highly expressed in lymphomas induced by a very virulent plus (vv+) GaHV-2 strain than those produced by a very virulent (vv) GaHV-2 strain, whereas the LAT-clustered miRNAs have equal expression [17] suggesting that the Meqclustered miRNAs may have a more significant role in GaHV-2 oncogenesis.

Up to now, only a few of the GaHV-2 miRNAs, such as miR-M4-5p [18-21], miR-M3-5p [22], and miR-M7-5p [23], have been shown to play critical roles in GaHV-2 replication, latency, pathogenesis, and/or oncogenesis. For most of the other viral miRNAs, their potential regulatory role in GaHV-2 biology remains speculative. In previous experiments [24], we have investigated the expression profiles of most viral miRNAs in very virulent GaHV-2 strain GX0101-challenged chickens during the late phases of disease and found that only a subset of GaHV-2 miRNAs was differentially expressed in diseased birds with obvious tissue-specific expression patterns. The course of MD is described in the standard 'Cornell Model' [25, 26]. This includes four phases: (1) the early cytolytic phase (2-7 dpi), (2) the latent phase (7-10 dpi onwards), (3) the late cytolytic phase (18 dpi onwards), and (4) the proliferative phase (28 dpi onwards). In the present study, we describe the expression profiles of all of the $26 \mathrm{GaHV}-2$ miRNAs during each stage in the cycle of MD pathogenesis, providing more information to help in unraveling their biological functions.
The animal experiments were conducted following the protocols of the Ethical and Animal Welfare Committee of Key Laboratory of Animal Immunology of the Ministry of Agriculture of China, simultaneously with those as we have previously reported [21]. Considering that our previous work [24] has shown that among eight different organs most GaHV-2 miRNAs are expressed at the highest levels in spleens, three birds challenged by GX0101 were randomly selected at $3,7,14,21,30,45$, and 60 days postinfection (dpi) for collecting the spleens and total RNAs were extracted using TRIzol reagent (Invitrogen) for subsequent experiments. Firstly, to evaluate the in vivo virus proliferation, gene expression levels of several representative GaHV-2 viral protein-coding genes in GX0101-infected chickens, including the unique oncogene meq (MDV EcoRI-Q), the $38 \mathrm{KD}$ phosphorylated protein gene (pp38), the structural glycoprotein B gene $(g B)$, and the immediateearly (IE) infected cell protein 4 gene (ICP4), have been measured by quantitative real-time PCR (qRT-PCR). For cDNA synthesis, aliquots of $100 \mathrm{ng}$ RNAs were first treated with RNase-free DNase I (TaKaRa) and then polyadenylated and reverse transcribed at $37{ }^{\circ} \mathrm{C}$ for $1 \mathrm{~h}$ in a $20 \mu \mathrm{l}$ reaction mixture, using the $\mathrm{NCode}^{\mathrm{TM}} \mathrm{VILO}^{\mathrm{TM}}$ miRNA cDNA Synthesis Kit (Invitrogen) according to the manufacturer's instructions. The relative quantification of GaHV-2 viral protein-coding genes was performed. Briefly, a total $20 \mu \mathrm{l}$ qPCR reaction was prepared, containing $10 \mu \mathrm{l}$ of SuperMix Universal, $5 \mathrm{ng}$ of cDNA, $50 \mathrm{nM}$ of ROX Reference Dye (Invitrogen), and $200 \mathrm{nM}$ of each primer specific for GaHV-2 genes (Table 1, primer pairs \#1-\#4). The 7500 Fast Real-Time PCR Systems (Applied Biosystems, Life Technologies, USA) was used for qPCR amplification with the reaction conditions set as follows: $95^{\circ} \mathrm{C}$ for $3 \mathrm{~min}, 40$ cycles of $95^{\circ} \mathrm{C}$ for $3 \mathrm{~s}$, and $60{ }^{\circ} \mathrm{C}$ for $30 \mathrm{~s}$, followed by the thermal denaturing step to generate the dissociation curves to verify amplification specificity. Relative quantification of the mRNA gene expression was standardized to the level of endogenous chicken glyceraldehyde phosphate dehydrogenase (GAPDH) transcript determined using the same qPCR conditions with primer pair \#5 (Table 1) and finally calculated with the $2^{-\Delta \Delta C_{t}}$ method. Secondly, the absolute quantitation of GaHV-2 miRNAs was performed by qPCR as previously described [27]. The primers \#6-\#31, as listed in Table 1, specific for each GaHV-2 miRNA, were used as the forward primers for the qPCR analysis of miRNA expression using the same reactions and conditions as for the amplifications of viral protein-coding genes. Each of $200 \mathrm{nM}$ of Universal qPCR Primer (Table 1, primer \#32), provided by the NCode $^{\mathrm{TM}}$ VILO $^{\mathrm{TM}}$ miRNA cDNA Synthesis Kit (Invitrogen), was used as the reverse primer. Synthetic miRNA miR-M4-5p (GenePharma, Shanghai, China) was used as 
Table 1 Primer pairs or primers used for the quantitative real-time PCR (qRT-PCR) analysis of the expression of viral miRNAs and GaHV-2 protein-coding genes in the present study

\begin{tabular}{|c|c|c|c|c|c|}
\hline No. & Primer (pair) & Target & Type $^{\mathrm{a}}$ & Sequence $\left(5^{\prime}-3^{\prime}\right)^{\mathrm{b}}$ & Length (nt) \\
\hline \multirow[t]{2}{*}{1} & qPCRMeq-F & meq & $5^{\prime}$ & CGCAGGAAGCAGACGGACTA & 20 \\
\hline & qPCRMeq-R & meq & $3^{\prime}$ & CCATAGGGCAAACTGGCTCAT & 21 \\
\hline \multirow[t]{2}{*}{2} & qMDV100-F & ICP4 & $5^{\prime}$ & GCACTGCATTCCGAGAGTCAT & 21 \\
\hline & qMDV100-R & ICP4 & $3^{\prime}$ & TTGGGAATTTGGAGGGCG & 18 \\
\hline \multirow[t]{2}{*}{3} & qPCRgB-F & $g B$ & $5^{\prime}$ & TCTAGGGCATGGCACACGAC & 20 \\
\hline & qPCRgB-R & $g B$ & $3^{\prime}$ & GAATACGGAAACACAGAGCGG & 21 \\
\hline \multirow[t]{2}{*}{4} & qPCRpp38-F & pp38 & $5^{\prime}$ & CCGAAAGACAAAACCCAAAT & 20 \\
\hline & qPCRpp38-R & pp38 & $3^{\prime}$ & ATGTAACCAGCATATAAGAACGC & 23 \\
\hline \multirow[t]{2}{*}{5} & qPCRGAPDH-F & $G A P D H$ & $5^{\prime}$ & AAGTCCCTGAAAATTGTCAGCAAT & 24 \\
\hline & qPCRGAPDH-R & $G A P D H$ & $3^{\prime}$ & ATGGCATGGACAGTGGTCATAAG & 23 \\
\hline 6 & miR-M1-3p-F & $\mathrm{miR}$ & $5^{\prime}$ & GCGCATGAAAGAGCGAAAA & 19 \\
\hline 7 & miR-M1-5p-F & $\mathrm{miR}$ & $5^{\prime}$ & TGTTCACTGTGCGGCAAAAA & 20 \\
\hline 8 & miR-M2-3p-F & $\operatorname{miR}$ & $5^{\prime}$ & CTGCCGCAGAATAGCTTAAAAA & 22 \\
\hline 9 & miR-M2-5p-F & $\mathrm{miR}$ & $5^{\prime}$ & GTTGTATTCTGCCCGGTAGTCC & 22 \\
\hline 10 & miR-M3-3p-F & $\operatorname{miR}$ & $5^{\prime}$ & GGGGGGTTCACATTTTTAAGTAAA & 24 \\
\hline 11 & miR-M3-5p-F & $\mathrm{miR}$ & $5^{\prime}$ & TGAAACCTCTCCCGCAAAAA & 20 \\
\hline 12 & miR-M4-3p-F & $\mathrm{miR}$ & $5^{\prime}$ & GGTTCTGACAGCATGACCAAAAA & 23 \\
\hline 13 & miR-M5-3p-F & $\operatorname{miR}$ & $5^{\prime}$ & TGTGTATCGTGGTCGTCTACTGTAAA & 26 \\
\hline 14 & miR-M4-5p-F & $\mathrm{miR}$ & $5^{\prime}$ & TTAATGCTGTATCGGAACCCTTC & 23 \\
\hline 15 & miR-M5-5p-F & $\mathrm{miR}$ & $5^{\prime}$ & CGTATGCGATCACATTGACAAAA & 23 \\
\hline 16 & miR-M6-3p-F & $\mathrm{miR}$ & $5^{\prime}$ & GATCCCTGCGAAATGACAGTAAA & 23 \\
\hline 17 & miR-M6-5p-F & $\mathrm{miR}$ & $5^{\prime}$ & TCTGTTGTTCCGTAGTGTTCTCAAA & 25 \\
\hline 18 & miR-M7-3p-F & $\mathrm{miR}$ & $5^{\prime}$ & TCGAGATCTCTACGAGATTACAGAAAA & 27 \\
\hline 19 & miR-M7-5p-F & $\mathrm{miR}$ & $5^{\prime}$ & CGGGGAGATCCCGATAAAAA & 20 \\
\hline 20 & miR-M8-3p-F & $\mathrm{miR}$ & $5^{\prime}$ & GTGACCTCTACGGAACAATAGTAAAAA & 27 \\
\hline 21 & miR-M8-5p-F & $\mathrm{miR}$ & $5^{\prime}$ & TATTGTTCTGTGGTTGGTTTCGA & 23 \\
\hline 22 & miR-M9-3p-F & $\operatorname{miR}$ & $5^{\prime}$ & CGAGGGCAGGAAAAAGAAAAA & 21 \\
\hline 23 & miR-M9-5p-F & $\operatorname{miR}$ & $5^{\prime}$ & CTTCCCCCCGGAGTTAAAAA & 20 \\
\hline 24 & miR-M10-3p-F & $\mathrm{miR}$ & $5^{\prime}$ & TCGAAATCTCTACGAGATAACAAAAA & 26 \\
\hline 25 & miR-M10-5p-F & $\mathrm{miR}$ & $5^{\prime}$ & TTGTCTCGTAGAGGTCCAGAAAAA & 24 \\
\hline 26 & miR-M11-3p-F & $\mathrm{miR}$ & $5^{\prime}$ & AGTTACATGGTCAGGGGATTAAAAA & 25 \\
\hline 27 & miR-M11-5p-F & $\mathrm{miR}$ & $5^{\prime}$ & TTTTCCTTACCGTGTAGCTTAGAAA & 25 \\
\hline 28 & miR-M12-3p-F & $\mathrm{miR}$ & $5^{\prime}$ & TGCATAATACGGAGGGTTCTAAAA & 24 \\
\hline 29 & miR-M12-5p-F & $\mathrm{miR}$ & $5^{\prime}$ & GCCCTCCGTATAATGTAAATGTAAAA & 26 \\
\hline 30 & miR-M13-3p-F & $\mathrm{miR}$ & $5^{\prime}$ & ATGGAAACGTCCTGGGAAAAA & 21 \\
\hline 31 & miR-M31-3p-F & $\mathrm{miR}$ & $5^{\prime}$ & CTACAGTCGTGAGCAGATCAAAAA & 24 \\
\hline 32 & Universal qPCR Primer & $\mathrm{miR}$ & $3^{\prime}$ & UA & \\
\hline
\end{tabular}

$m i R$ miRNA, GAPDH glyceraldehyde phosphate dehydrogenase, $m e q \underline{\mathrm{MDV}} \underline{E} c o \mathrm{RI}-\underline{\mathrm{Q}}$, ICP4 immediate-early gene ICP4 (infected cell protein 4), $g B$ virion membrane glycoprotein $\mathrm{B}$, pp $3838 \mathrm{KD}$ phosphorylated protein

a $5^{\prime}$, forward primer; $3^{\prime}$, reverse primer

${ }^{b}$ UA, the Universal qPCR Primer is provided by Invitrogen with unavailable sequence

the standard to estimate the absolute miRNA copy number. Each reaction was repeated in triplicate and the values were used to calculate the means $(\mathrm{M}) \pm$ standard deviations (SD), utilizing the software GraphPad Prism (version 5.0). The differences in expression levels of each viral protein-coding gene or miRNA between groups determined at distinct time point post-challenge were compared to $3 \mathrm{dpi}$ and analyzed by one-way analysis of variance (One-way ANOVA, LSD) 
and were considered significant between groups at a probability level of $p<0.05$.

Using the pattern of disease progress described in the 'Cornell Model' [25, 26], the GaHV-2 protein-coding genes, including $g B, I C P 4, p p 38$, and $m e q$, gave expression patterns as expected in the spleens of GX0101-challenged birds during the early stages of GaHV-2 infection (Fig. 1). The relative expression levels of all four viral genes increased first at $7 \mathrm{dpi}$ (the early cytolytic phase), decreased at $14 \mathrm{dpi}$ (the latent phase), and then peaked at 21 dpi (the late cytolytic phase). At the late time period of 30-60 dpi (the transformation and proliferative phase), the expressions of the genes of $g B, I C P 4$, and $p p 38$ were gradually reduced (Fig. 1a-c) but meq remained expressed at a higher level (Fig. 1d), reflecting its role as a viral oncogene [26].

However, distinct from the viral protein-coding genes, the GaHV-2 miRNAs could be divided into three groups with different temporal expression profiles during the progress of the disease. The expression levels of 12 viral miRNAs, including the miR-M4-5p, miR-M2-5p, miR-M23p, miR-M5-3p, miR-M3-3p, and miR-M12-3p in the Meqcluster (Fig. 2a); the miR-M8-5p, miR-M8-3p, miR-M65p, miR-M6-3p, and miR-M7-3p in the LAT-cluster (Fig. 2b); and the miR-M11-5p in the Mid-cluster (Fig. 2c) increased and reached a first peak at 14-21 dpi, decreased by $30 \mathrm{dpi}$, and were then highly expressed again at 45-60 dpi. These miRNAs constitute the first group and they displayed an expression pattern that may be closely correlated to the latent, late cytolytic, and/or the proliferative phases in MD pathogenesis. One of the highest expressed GaHV-2 miRNAs, miR-M4-5p, has been characterized as a functional miR-155 ortholog [18], and deletion of miR-M4 from the viral genomes of the virulent (v) GaHV-2 strain pRB-1B5 and the very virulent GaHV-2 strain GX0101 significantly decrease their oncogenicity $[20,21]$. In addition to the previously reported conserved cellular genes targeted by cellular miR-155 [28], we have shown recently that miR-M4-5p down-regulates the expression of the latent transforming growth factor- $\beta$ (TGF$\beta$ )-binding protein 1 (LTBP1). This leads to a significant decrease in activation and secretion of TGF- $\beta 1$, with concomitant suppression of TGF- $\beta$ signaling and a significant increase in the level of expression of c-MYC, a well-known oncogene which is critical for the virally induced tumorigenesis [29]. In addition to its involvement in GaHV-2 oncogenesis, miR-M4-5p has also been shown to specifically inhibit the production of UL28 [19], a viral protein homologous to a human herpesvirus 1 (HHV-1) protein involved in the cleavage/packaging of viral DNA. These facts help to explain the expression pattern of miRM4-5p during GaHV-2's life cycle and in particular its higher expression level during the late stages of the developing disease. The other 11 miRNAs of group I displayed expression patterns similar to that of miR-M4-5p. We have recently shown that in addition to miR-M4-5p, deletions of the other individual Meq-clustered miRNAs, i.e., miR-M2, miR-M3, and miR-M12, also variably
Fig. 1 Relative expression levels of the GaHV-2 proteincoding genes analyzed by qRTPCR. a $g B$, the gene of glycoprotein $\mathrm{B}$; b ICP4, the gene of infected cell protein 4 ; c pp38, the gene of $38 \mathrm{KD}$ phosphorylated protein; $\mathbf{d} M e q$, the oncogene of MDV EcoRI-Q. The endogenous chicken $G A P D H$ gene was used as a standard and the relative quantification of mRNA expression was calculated with the $2^{-\Delta \Delta C_{t}}$ method. Columns represent the mean of relative expression levels in the spleens of three randomly selected birds, determined in triplicate. Error bars indicate $1 \times \mathrm{SD}$. The star indicates significant difference $(p<0.05)$ compared to that determined at $3 \mathrm{dpi}$
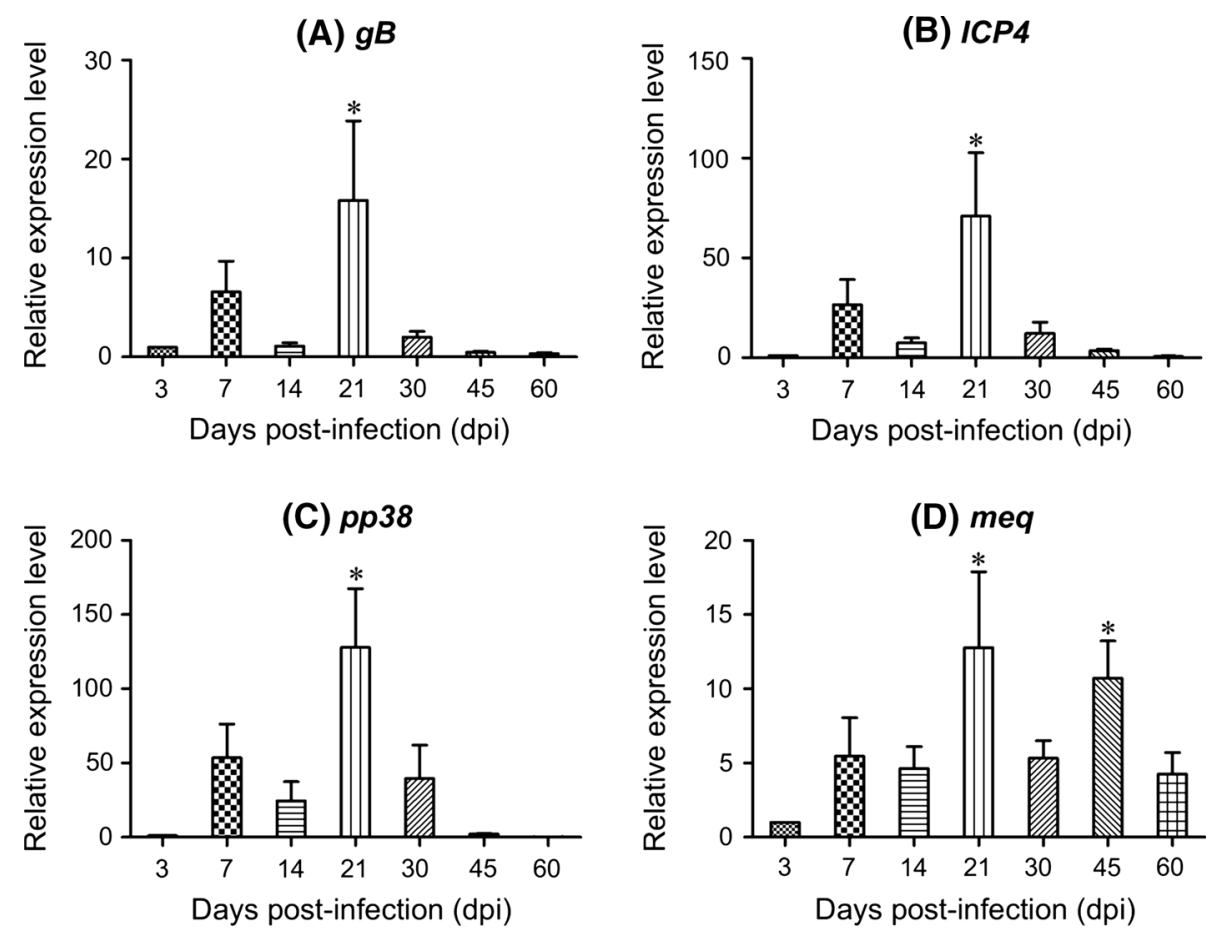


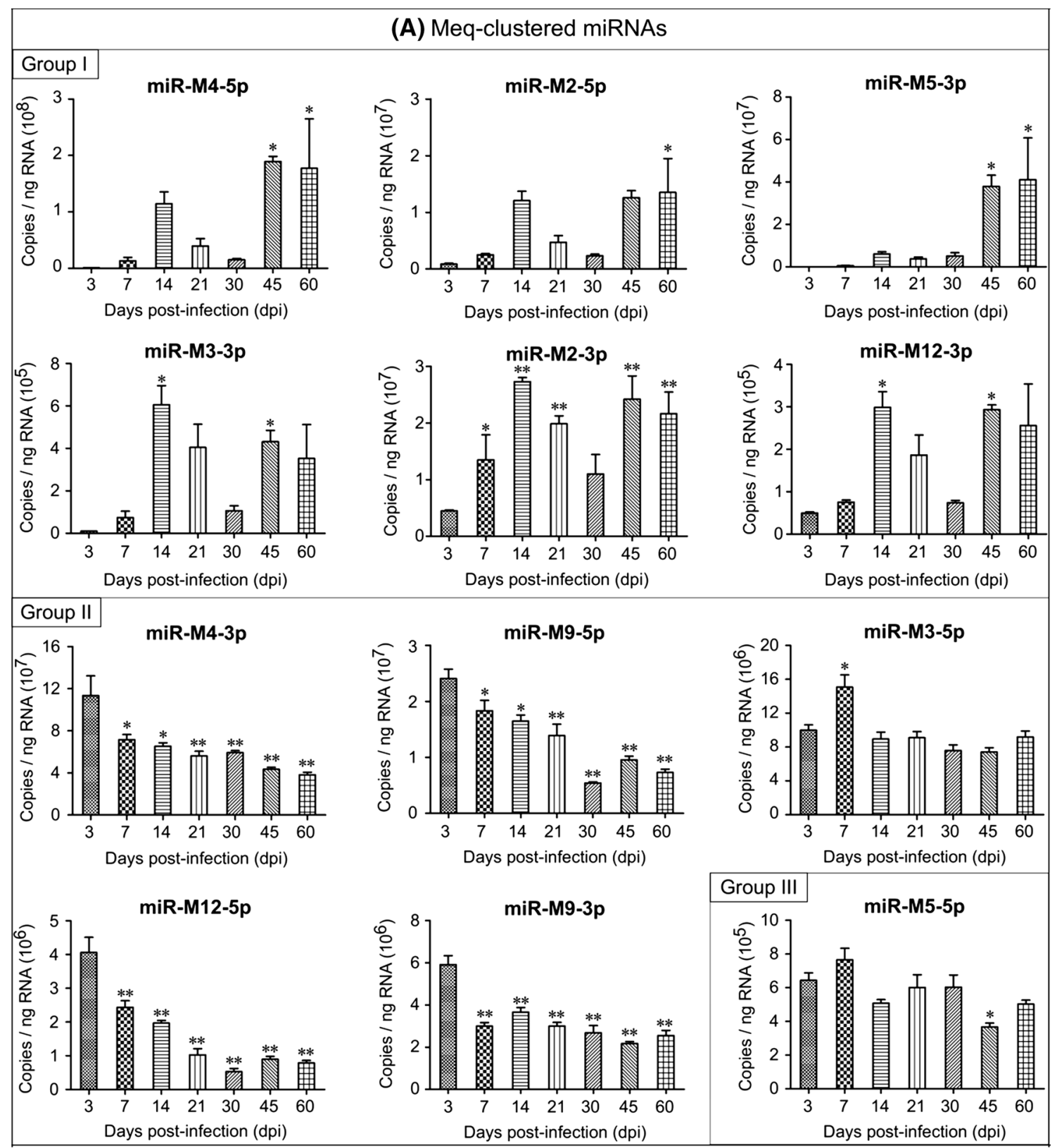

Fig. 2 Expression profiles of GaHV-2 miRNAs during the different phases of the developing disease. a The Meq-clustered miRNAs; b the LAT-clustered miRNAs; c the Mid-clustered miRNAs. A quantitative real-time PCR was performed to determine miRNA expression and the absolute copy number was estimated using

decrease the pathogenicity and oncogenicity of the virus [30]. Thus, whether or not these miRNAs encoded in the Meq or other two clusters play a similar part in the virus life cycle remains to be further studied.

The other 12 GaHV-2 miRNAs showing a different expression pattern include miR-M4-3p, miR-M12-5p, miRM9-5p, miR-M9-3p, and miR-M3-5p in the Meq-cluster (Fig. 2a); the miR-M7-5p, miR-M10-5p, miR-M10-3p, and miR-M13-3p in the LAT-cluster (Fig. 2b); and the miR- synthetic miR-M4-5p as the standard. Columns represent the mean miRNA expression levels in the spleens from three randomly selected birds, determined in triplicate. Error bars indicate $1 \times$ SD. The single and double stars indicate significant differences $(p<0.05$ or $p<0.01)$ compared to that determined at $3 \mathrm{dpi}$

M1-3p, miR-M11-3p, and miR-M31-3p in the Mid-cluster (Fig. 2c) and constitute the second group. Most of these miRNAs were expressed at higher levels during the first 1-2 weeks post-challenge with subsequent low levels of expression, although some display a slight increase at 45 or 60 dpi. Based on these results, it seems that the miRNAs included in group II are more closely correlated to the early cytolytic and/or the latent phases in GaHV-2's life cycle. Such a suggestion may be further supported in part by 

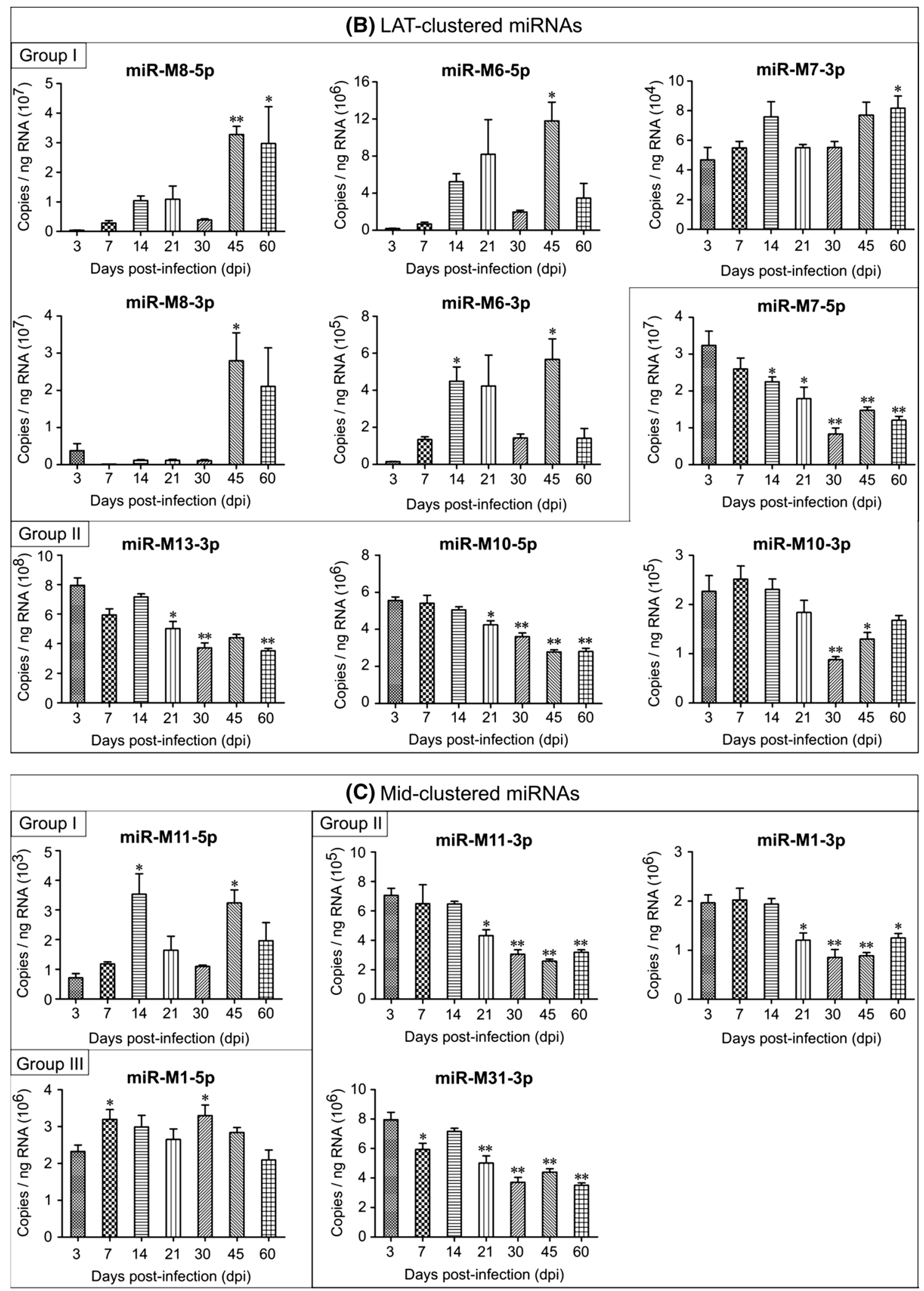

Fig. 2 continued 
some recent reports. The miR-M4-3p has been shown to specifically target and repress UL32 production [19], another viral protein homologous to a HHV-1 protein involved in the cleavage/packaging of herpes virus DNA. A recent study has also demonstrated that miR-M7-5p, located in the first intron of the latency-associated transcripts (LATs), targets the immediate-early genes ICP4 and ICP27 [23], providing a possibly contribution to the establishment and/or maintenance of latency. Furthermore, the miR-M3$5 \mathrm{p}$, which was highly expressed particularly at $7 \mathrm{dpi}$, has been shown to target and down-regulate the expression of Smad2 [22], a critical component in TGF- $\beta$ signal pathway, potentially creating a cellular environment beneficial to viral latency. Thus, the possibility of similar roles for the other GaHV-2 miRNAs of group II may be uncovered in future studies.

However, the other two miRNAs, miR-M5-5p in the Meq-cluster (Fig. 2a) and miR-M1-5p in the Mid-cluster (Fig. 2c), display no identical expression features, constitute a third group (group III). Previous studies have shown that the transcription of GaHV-2 miRNAs located in both of the Meq- and Mid-clusters is driven by a single promoter 'prmiRM9M4' [13], whereas that of all the LAT-clustered miRNAs are driven by a p53-dependent promoter [14]. It is interesting that during the developing of disease, some of the mature miRNAs processed from a same pre-miRNAs (such as miR-M2, miR-M9, miR-M6, miR-M8, and miRM10) display similar expression profiles, while the other miRNAs represent expression patterns different from each other. Such a phenomenon has also been observed among miRNAs that had been identified in diverse species, such as in animals, plants, and even in viruses, but little of the underlined precious mechanism is known to date. In addition, we also observed that the expression patterns of miR-M9-5p and miR-M7-5p presented here were not in accordance with our previous report [24]. Whether it was caused by the differential methodology, primer or other factors need to be further studied.

In recent years, identifications of miRNA expression signatures in many cancers have increased our further understanding of the connection between miRNA and cancer [31]. Our present research on the in vivo expression profiles of GaHV-2 miRNAs may provide meaningful clues for the prediction, selection, and characterization of their mRNA targets, and further evaluating their biological functions in the virus life cycle and development of MD tumors and other cancers. Based on the successful establishment of strategies for mRNA target prediction and subsequent experimental identification along with the application of bacterial artificial chromosomes (BAC) in research on MD pathogenesis, focus on the miRNAs highlighted in this study should reveal more about their roles.
Acknowledgments This work was supported by Grants of the National Natural Science Foundation of China (No. 31372445), the Key Project of NSFC-Guangdong Joint Fund (No. U1131005), and the Science-Technology Foundation for Outstanding Young Scientists of Henan Academy of Agricultural Sciences (No. 2013YQ28). The authors gratefully acknowledge the critical review by Prof. Norman A. Gregson (ION, UCL, London, UK).

Open Access This article is distributed under the terms of the Creative Commons Attribution License which permits any use, distribution, and reproduction in any medium, provided the original author(s) and the source are credited.

\section{References}

1. A.J. Davison, R. Eberle, B. Ehlers, G.S. Hayward, D.J. McGeoch, A.C. Minson, P.E. Pellett, B. Roizman, M.J. Studdert, E. Thiry, The order Herpesvirales. Arch. Virol. 154, 171-177 (2009)

2. R.L. Witter, K. Schat, Marek's disease, in Diseases of Poultry, 11th. Edition edn., ed. by Y.M. Saif (Iowa State University Press, Ames: 2003), pp. 407-464

3. N. Osterrieder, J.P. Kamil, D. Schumacher, B.K. Tischer, S. Trapp, Marek's disease virus: from miasma to model. Nat. Rev. Microbiol. 4, 283-294 (2006)

4. A. Kozomara, S. Griffiths-Jones, miRBase: integrating microRNA annotation and deep-sequencing data. Nucleic Acids Res. 39, D152-D157 (2011)

5. I.W. Boss, K.B. Plaisance, R. Renne, Role of virus-encoded microRNAs in herpesvirus biology. Trends Microbiol. 17, 544-553 (2009)

6. R.P. Kincaid, C.S. Sullivan, Virus-encoded microRNAs: an overview and a look to the future. PLoS Pathog. 8, e1003018 (2012)

7. J. Burnside, E. Bernberg, A. Anderson, C. Lu, B.C. Meyers, P.J. Green, N. Jain, G. Isaacs, R.W. Morgan, Marek's disease virus encodes MicroRNAs that map to meq and the latency-associated transcript. J. Virol. 80, 8778-8786 (2006)

8. Y. Yao, Y. Zhao, H. Xu, L.P. Smith, C.H. Lawrie, M. Watson, V. Nair, MicroRNA profile of Marek's disease virus-transformed T-cell line MSB-1: predominance of virus-encoded microRNAs. J. Virol. 82, 4007-4015 (2008)

9. L.A. Waidner, R.W. Morgan, A.S. Anderson, E.L. Bernberg, S. Kamboj, M. Garcia, S.M. Riblet, M. Ouyang, G.K. Isaacs, M. Markis, B.C. Meyers, P.J. Green, J. Burnside, MicroRNAs of Gallid and Meleagrid herpesviruses show generally conserved genomic locations and are virus-specific. Virology 388, 128-136 (2009)

10. Y. Yao, Y. Zhao, H. Xu, L.P. Smith, C.H. Lawrie, A. Sewer, M. Zavolan, V. Nair, Marek's disease virus type 2 (MDV-2)-encoded microRNAs show no sequence conservation with those encoded by MDV-1. J. Virol. 81, 7164-7170 (2007)

11. Y. Yao, Y. Zhao, L.P. Smith, M. Watson, V. Nair, Novel microRNAs (miRNAs) encoded by herpesvirus of Turkeys: evidence of miRNA evolution by duplication. J. Virol. 83, 6969-6973 (2009)

12. J. Luo, M. Teng, J.M. Fan, F.Y. Wang, L. Zhou, R.G. Deng, G.P. Zhang, Marek's disease virus-encoded microRNAs: genomics, expression and function. Sci. China Life Sci. 53, 1174-1180 (2010)

13. D. Coupeau, G. Dambrine, D. Rasschaert, Kinetic expression analysis of the cluster mdv1-mir-M9-M4, genes meq and vIL-8 differs between the lytic and latent phases of Marek's disease virus infection. J. Gen. Virol. 93, 1519-1529 (2012) 
14. G. Stik, S. Laurent, D. Coupeau, B. Coutaud, G. Dambrine, D. Rasschaert, B. Muylkens, A p53-dependent promoter associated with polymorphic tandem repeats controls the expression of a viral transcript encoding clustered microRNAs. RNA 16, 2263-2276 (2010)

15. D.P. Bartel, MicroRNAs: genomics, biogenesis, mechanism, and function. Cell 116, 281-297 (2004)

16. R.W. Carthew, E.J. Sontheimer, Origins and mechanisms of miRNAs and siRNAs. Cell 136, 642-655 (2009)

17. R. Morgan, A. Anderson, E. Bernberg, S. Kamboj, E. Huang, G. Lagasse, G. Isaacs, M. Parcells, B.C. Meyers, P.J. Green, J. Burnside, Sequence conservation and differential expression of Marek's disease virus microRNAs. J. Virol. 82, 12213-12220 (2008)

18. Y. Zhao, Y. Yao, H. Xu, L. Lambeth, L.P. Smith, L. Kgosana, X. Wang, V. Nair, A functional MicroRNA-155 ortholog encoded by the oncogenic Marek's disease virus. J. Virol. 83, 489-492 (2009)

19. B. Muylkens, D. Coupeau, G. Dambrine, S. Trapp, D. Rasschaert, Marek's disease virus microRNA designated Mdv1-pre-miR-M4 targets both cellular and viral genes. Arch. Virol. 155, 1823-1837 (2010)

20. Y. Zhao, H. Xu, Y. Yao, L.P. Smith, L. Kgosana, J. Green, L. Petherbridge, S.J. Baigent, V. Nair, Critical role of the virusencoded microRNA-155 ortholog in the induction of Marek's disease lymphomas. PLoS Pathog. 7, e1001305 (2011)

21. Z.H. Yu, M. Teng, A.J. Sun, L.L. Yu, B. Hu, L.H. Qu, K. Ding, X.C. Cheng, J.X. Liu, Z.Z. Cui, G.P. Zhang, J. Luo, Virusencoded miR-155 ortholog is an important potential regulator but not essential for the development of lymphomas induced by very virulent Marek's disease virus. Virology 448, 55-64 (2014)

22. S. Xu, C. Xue, J. Li, Y. Bi, Y. Cao, Marek's disease virus type 1 microRNA miR-M3 suppresses cisplatin-induced apoptosis by targeting Smad2 of the transforming growth factor beta signal pathway. J. Virol. 85, 276-285 (2011)

23. S. Strassheim, G. Stik, D. Rasschaert, S. Laurent, Mdv1-miR-M7$5 \mathrm{p}$, located in the newly identified first intron of the latency-associated transcript of Marek's disease virus, targets the immediateearly genes ICP4 and ICP27. J. Gen. Virol. 93, 1731-1742 (2012)

24. J. Luo, A.J. Sun, M. Teng, H. Zhou, Z.Z. Cui, L.H. Qu, G.P. Zhang, Expression profiles of microRNAs encoded by the oncogenic Marek's disease virus reveal two distinct expression patterns in vivo during different phases of disease. J. Gen. Virol. 92, 608-620 (2011)

25. B.W. Calnek, Pathogenesis of Marek's disease virus infection. Curr. Topics Microbiol. Immunol. 255, 25-55 (2001)

26. S.J. Baigent, F. Davison, Marek's disease virus: biology and life cycle, in Marek's Disease, An Evolving Problem, ed. by F. Davison, V. Nair (Elsevier Academic Press, Oxford, 2004), pp. $62-77$

27. R. Shi, V.L. Chiang, Facile means for quantifying microRNA expression by real-time PCR. Biotechniques 39, 519-525 (2005)

28. O. Parnas, D.L. Corcoran, B.R. Cullen, Analysis of the mRNA targetome of microRNAs expressed by Marek's disease virus. MBio 5, e01060-13 (2014)

29. J.Q. Chi, M. Teng, Z.H. Yu, H. Xu, J.W. Su, P. Zhao, G.X. Xing, H.D. Liang, R.G. Deng, L.H. Qu, G.P. Zhang, J. Luo, Marek's disease virus-encoded analog of microRNA-155 activates the oncogene c-Myc by targeting LTBP1 and suppressing the TGF- $\beta$ signaling pathway. Virology 476, 72-84 (2015)

30. M. Teng, Z.H. Yu, A.J. Sun, Y.J. Min, J.Q. Chi, P. Zhao, J.W. Su, Z.Z. Cui, G.P. Zhang, J. Luo, The significance of the individual Meq-clustered miRNAs of Marek's disease virus in oncogenesis. J. Gen. Virol. (2014). doi:10.1099/jgv.0.000013

31. G.A. Calin, C.M. Croce, MicroRNA signatures in human cancers. Nat. Rev. Cancer 6, 857-866 (2006) 